\title{
Variation principle in calculating the flow of a two-phase mixture in the pipes of the cooling systems in high-rise buildings
}

\author{
Andrey Aksenov ${ }^{1}$, and Anna Malysheva ${ }^{1 *}$ \\ ${ }^{1}$ Moscow State University of Civil Engineering, 12933, Moscow, Yaroslavskoe shosse, 26, Russia
}

\begin{abstract}
The analytical solution of one of the urgent problems of modern hydromechanics and heat engineering about the distribution of gas and liquid phases along the channel cross-section, the thickness of the annular layer and their connection with the mass content of the gas phase in the gasliquid flow is given in the paper.The analytical method is based on the fundamental laws of theoretical mechanics and thermophysics on the minimum of energy dissipation and the minimum rate of increase in the system entropy, which determine the stability of stationary states and processes. Obtained dependencies disclose the physical laws of the motion of two-phase media and can be used in hydraulic calculations during the design and operation of refrigeration and air conditioning systems.
\end{abstract}

\section{Introduction}

Questions related to the distribution of a two-phase medium in a stream, i.e. with the true volumetric gas content (the relative cross-sectional area occupied by the gaseous medium) in the gas-liquid flow, and also with the slip coefficient (ratio of the average phase velocities) are key in the hydrodynamics of two-phase flows, for example, in the study of steam flow in air conditioning systems of industrial civil tall buildings .

Among the numerous studies in this area, experimental methods predominate, and in the calculations, empirical formulas and nomograms are mainly used.

When analyzing a two-phase flow the Fauske, Zivi relation can be used $[1,2]$ :

$$
\begin{aligned}
& \frac{\overrightarrow{w_{g}}}{\overline{w_{l}}}=\left(\frac{\rho_{l}}{\rho_{g}}\right)^{\frac{1}{2}}, \\
& \frac{\overrightarrow{w_{g}}}{\overrightarrow{w_{l}}}=\left(\frac{\rho_{l}}{\rho_{g}}\right)^{\frac{1}{3}},
\end{aligned}
$$

where $w_{\mathrm{g}}$ - gas phase velocity; $w_{\mathrm{l}}$ - liquid phase velocity; $\rho_{\mathrm{l}}$ - liquid density; $\rho_{\mathrm{g}}-$ gas density.

The first of these formulas is derived from the minimum of the total amount of motion of the liquid and gas phases in a two-phase flow. These formulas do not reflect the influence on the slip coefficient of either the mass content of gas or the physical properties of the liquid

\footnotetext{
*Corresponding author: mani495@mail.ru
} 
and gaseous media (except for the ratio of their densities), which limits the possibility of their application.

In the present work, it is made an attempt to solve the problem of the volume gas content and the phase slip coefficient applied to two-phase annual flows on the basis of the principle of minimum energy dissipation in a stabilized flow associated with irreversible losses by internal friction during the motion of real media.

To simplify the analysis, the annular structure of the adiabatic two-phase flow is adopted as the design model. The solution of the problem of the relative area occupied by the gas nucleus $\varphi$ and the corresponding phase slip coefficient $s=\left(\overline{w_{g}} / \overline{w_{l}}\right)$ in a symmetric horizontal annular flow, depending on the determining parameters.

From the thermodynamic point of view, in the flow of a viscous liquid degradation occurs (energy depreciation), as in all other real processes taking place in nature, the characteristic feature of which is the increase in the entropy of the system. Internal nonequilibrium processes always act in a direction that causes a decrease in the rate of entropy increase [3, 4]. In the thermodynamics of irreversible processes, this law is formulated as follows: as the system goes over to the stationary-nonequilibrium state, the value of the incremental increase in entropy decreases, and when the stationary-nonequilibrium state is reached, it is of the smallest value compatible with external constraints [5].

\section{Methods}

Let us consider the annular regime of a stabilized gas-liquid flow in a pipe with radius of $R_{0}$, shown in the Fig. 1. This flow will consist of a ring layer of liquid with thickness $\delta$ and a cylindrical gas core with radius $R_{G}=R_{0}-\delta$. The mass flow of fluid $G_{l}$ and gas $G_{g}$ and their physical parameters (density, viscosity) $\rho_{g} \rho_{l} \mu_{g} \mu_{l}$ are assumed to be known

Let us select a cylindrical element of radius $\mathrm{R}$ of unit length in the course of the flow. On the surface of the selected element, the force of internal friction acts. It is equal to

where $\tau$ - tangential friction stress, $\mathrm{N} / \mathrm{m}^{2}$.

$$
\mathrm{T}=\tau 2 \pi R_{1}
$$

The second work of the internal friction force is equal to the product of the value of $\tau$ by the rate of shear deformation on this axis:

$$
d E=2 \pi R \tau \frac{d w}{d R} d R
$$

The second work of internal friction along the entire section of the gas-liquid flow is expressed by the sum of the integrals:

$$
E=2 \pi \int_{0}^{R_{0}-\delta} \tau_{\Gamma} \cdot R \frac{d w_{g}}{d R} \cdot d R+2 \pi \int_{R_{0}-\delta}^{R_{0}} \tau_{l} \cdot R \frac{d w_{l}}{d R} \cdot d R
$$

In this case the first term $E_{g}$ refers to the gas nucleus, and the second one $E_{l}$ to the annular layer of the liquid; $E=E_{g}+E_{l}$; $\tau_{g}$ и $\tau_{l}$ - tangential friction constraint in the gas and liquid phases of the flow; $\mathrm{N} / \mathrm{m}^{2} ; w_{g}$ и $w_{l}$ - velocity of the gas and liquid phases on the surface of radius $\mathrm{R}$ in the cross section, $\mathrm{m} / \mathrm{s}$.

The value E expresses the second amount of dissipation in the cross section of the flow over a section of unit length.

Taking into account the law on the minimum rate of entropy increase in stationary irreversible processes or the variation principle of least action known in classical mechanics, we can conclude that the distribution of the gas and liquid phases along the cross section will be such that for any given mass flow gas content and other defining flow parameters, the second work of internal friction forces is minimal. It ensures the stability of stationary flows. Proceeding from this, it is possible to establish the relative cross-sectional area occupied by 
the gas phase $\varphi_{\mathrm{K}}=\frac{F_{g}}{F}$ (true volume gas content) and the ratio between the average velocities of the gas and liquid phases $\overline{w_{g}} / \overline{w_{l}}$. [6]

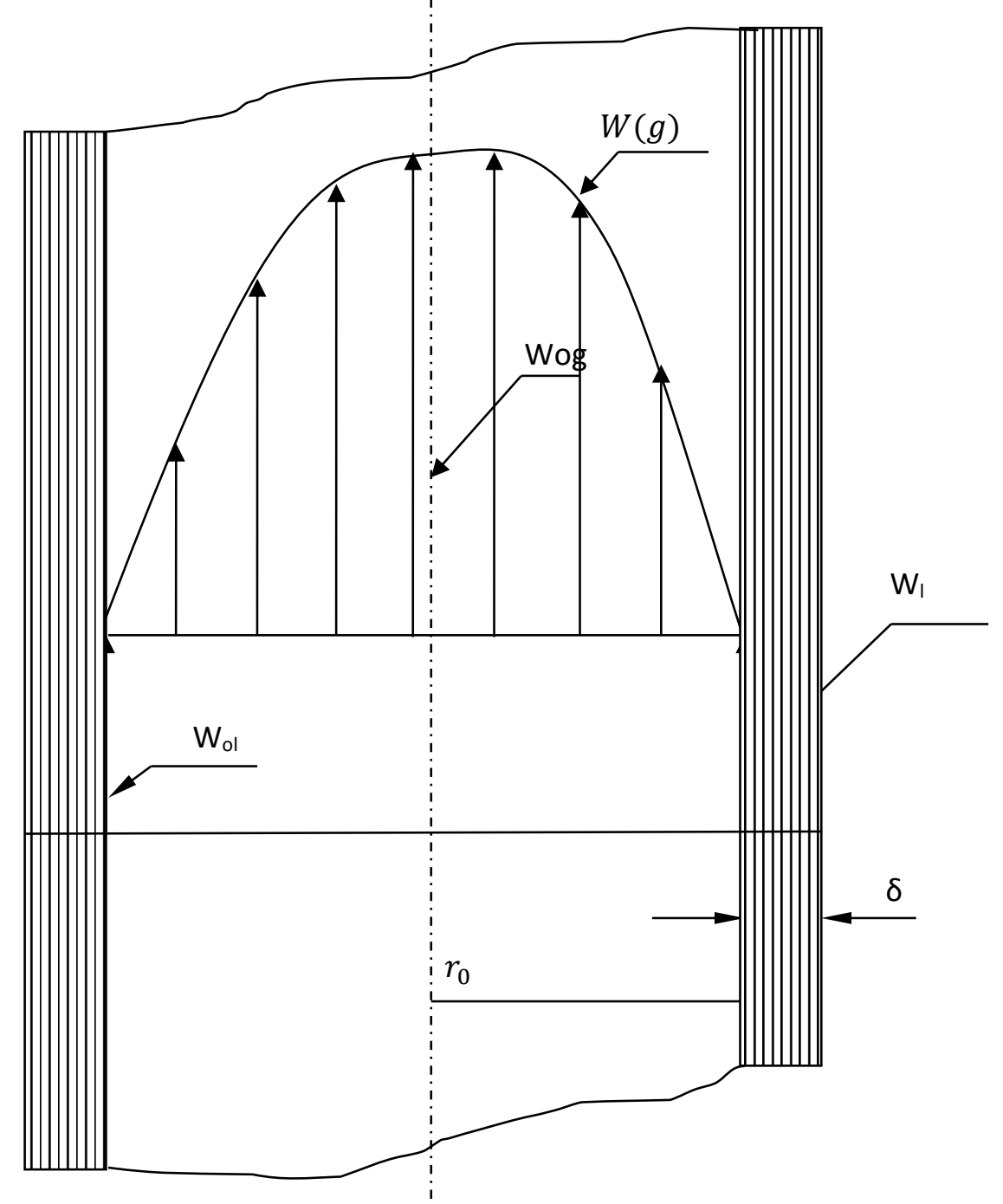

Fig. 1. Calculation model for the flow of a two-phase annular gas-liquid flow

We consider the problem posed for various

\subsection{Laminar flow in the gas nucleus and in the liquid layer}

For laminar flow, the magnitude of the tangential frictional stress is expressed by Newton's law:

where $y=R_{0}-R$.

$$
\tau=\mu \frac{d w}{d y}
$$

Consequently, the integral (3) can be rewritten in the following form:

$$
E=2 \pi \mu_{g} \int_{0}^{R_{0}-\delta} R \cdot\left(\frac{d w_{g}}{d R}\right)^{2} \cdot d R+2 \pi \mu_{l} \int_{R_{0}-\delta}^{R_{0}} R \cdot\left(\frac{d w_{l}}{d R}\right)^{2} \cdot d R .
$$


where $\mu_{g}$ и $\mu_{l}$ - dynamic viscosity coefficients of gas and liquid media, Pa.s.

The law of distribution of the phase velocities over the cross section of the flow will be assumed to be parabolic in the gas nucleus and linear in the liquid ring layer:

$$
w_{g}=w_{0 g}\left[1-\left(\frac{R}{R_{0}-\delta}\right)^{2}\right]+w_{0 l},
$$

where $\omega_{0 g}$ - relative velocity on the axis of the gas nucleus, $\mathrm{m} / \mathrm{s}$;

$$
\begin{array}{r}
0 \leq R \leq R_{g} ; R_{g}=R_{0}-\delta ; \\
w_{l}=w_{0 l} \frac{R_{0}-R}{\delta} ;
\end{array}
$$

where $R_{g} \leq R \leq R_{0}$.

According to the formula (5)

$$
\left(\frac{d w_{g}}{d R}\right)^{2}=\frac{4 w_{0 g}^{2} \cdot R^{2}}{\left(R_{0}-\delta\right)^{4}}
$$

Consequently

$$
E=\frac{2 \pi \mu_{g}-w_{0 g}^{2}}{\left(R_{0}-\delta\right)^{4}} \int_{0}^{R_{0}-\delta} R^{3} \cdot d R=2 \pi \mu_{g} w_{0 g}^{2} .
$$

Since the relative velocity on the $\omega_{0 \text { r }}$ axis is related to the average relative velocity in the nucleus by the relation $w_{0 g}=2 \overline{w_{g}}$, then

$$
E_{g}=8 \pi \mu_{g} w_{g}^{2}
$$

where

$$
\overline{w_{g}}=\overline{w_{g(a b s)}}-\omega_{0 l} .
$$

Let us find the relationship between the maximum $w_{0 l}$ and the average velocities in the section of the liquid phase. To do this, we calculate the integral

$$
\overline{w_{l}}=\frac{2 \pi \int_{R_{0}-\delta}^{R_{0}} \rho_{l} R w_{l} d R}{\pi \rho_{l}\left[R_{0}^{2}-\left(R_{0}-\delta\right)^{2}\right]}=\frac{2}{\left(2 R_{0}-\delta\right) \delta} \int_{R_{0}-\delta}^{R_{0}} R w_{l} d R .
$$

Taking into account relation (6), we will obtain

$$
\overline{w_{l}}=\frac{2 w_{0 l}}{\left(2 R_{0}-\delta\right) \delta^{2}} \int_{R_{0}-\delta}^{R_{0}} R\left(R_{0}-R\right) d R=\frac{w_{0 l}(3-2 \eta)}{3(2-\eta)},
$$

where $\eta$ - the relative thickness of the annular liquid layer; $\eta=\frac{\delta}{R_{0}}$.

Consequently

$$
w_{0 l}=3 \overline{w_{l}} \frac{2-\eta}{3-2 \eta}
$$

Taking into account continuity:

$$
w_{0 l}=\frac{3 G_{l}}{\pi \rho_{l} R_{0}^{2}(3-2 \eta)} .
$$

Average absolute velocity of the gaseous medium

$$
\overline{W_{g(a b s)}}=\frac{G_{g}}{\pi \rho_{g} R_{0}^{2}(1-\eta)^{2}},
$$

where $G_{l}$ и $G_{g}$ - mass flow of fluid and gas in the flow, $\mathrm{kg} / \mathrm{s}$. 
Taking into account formulas (12) and (13), expression (8) can be rewritten in the following form:

$$
\begin{array}{r}
E_{g}=\frac{8 \mu_{g} G^{2}(1-x)^{2}}{\pi R_{0}^{4} \rho_{l}^{2}} \cdot\left[\frac{\mathrm{A}}{(1-\eta)^{2}}-\frac{3}{\eta(2-3 \eta)}\right]^{2}, \\
\quad \text { where } \mathrm{A}=\frac{x}{1-x} \cdot\left(\frac{\rho_{l}}{\rho_{g}}\right) ; \quad G=G_{g}+G_{l} ;
\end{array}
$$

$x$ - mass flow rate of the gas phase in the flow.

Let us find the second term of the expression (4). According to the formula (6)

$$
E_{l}=2 \pi \mu_{l} \int_{R_{0}-\delta}^{R_{0}} R\left(\frac{w_{0 l}}{\delta}\right)^{2} d R=\pi \mu_{l} \omega_{0 l}^{2} \frac{(2-\eta)}{\eta} .
$$

Taking into account formula (12)

$$
E_{l}=\frac{9 \mu_{l} G^{2}(1-x)^{2}(2-\eta)}{\pi \rho_{l}^{2} R_{0}^{4} \eta^{3}(3-2 \eta)^{2}} .
$$

Thus, the sum (14) and (17) will be equal to:

$$
E_{l}=\frac{8 \mu_{g} G^{2}(1-x)^{2}}{\pi R_{0}^{4} \rho_{l}^{2}} \cdot\left\{\left[\frac{\mathrm{A}}{(1-\eta)^{2}}-\frac{3}{\eta(2-3 \eta)}\right]^{2}+\frac{9 \mu_{l} G^{2}(1-x)^{2}(2-\eta)}{\pi \rho_{l}^{2} R_{0}^{4} \eta^{3}(3-2 \eta)^{2}}\right\}
$$

It is easy to show that $(1-\eta)^{2}=\frac{F_{g}}{F}=\varphi$;

$$
G=\pi R_{0}^{2}(\rho w)
$$

where $\rho w$ - mass velocity of the gas-liquid mixture, $\mathrm{kg} / \mathrm{m}^{2} \cdot \mathrm{s}$.

Therefore, expression (18) can be written in the following form:

$$
E=\frac{8 \pi \mu_{g}(\rho w)^{2}(1-x)^{2}}{\rho_{l}}\left\{\left[\frac{A}{\varphi}-\frac{3}{(1-\sqrt{\varphi})(1+2 \sqrt{\varphi})}\right]^{2}+\frac{9 \mu_{l}(1+\sqrt{\varphi})}{8 \mu_{g}(1-\sqrt{\varphi})^{3}(1+2 \sqrt{\varphi})^{2}}\right\} .
$$

Let us find the required value of the true volume gas content $\varphi_{\text {ст }}$ and the relative thickness of the liquid annual layer $\eta$ (in the stationary flow) from the condition of a minimum of the value $E$ as a function of the argument $\varphi(19)$ or the argument $\eta(18)$.

The necessary condition for the extremum:

$\left(\frac{\partial E}{\partial \varphi}\right)_{\varphi=\varphi_{s t}}=0$, or $\left(\frac{\partial E}{\partial \eta}\right)_{\eta=\eta_{s t}}=0$.

The nature of the functions makes it possible not to justify sufficient conditions for a minimum.

For the sake of simplicity, we do not write the index "st" for $\eta$ and $\varphi$ in the sequel.

Let us find the derivative:

$$
\begin{aligned}
& \frac{\partial}{\partial \eta}\left\{\left[\frac{A}{(1-\eta)^{2}}-\frac{3}{\eta(3-2 \eta)}\right]-\frac{9 \mu_{l}(2-\eta)}{8 \mu_{g} \eta^{3}(3-2 \eta)^{2}}\right\}=2\left[\frac{A}{(1-\eta)^{2}}-\frac{3}{\eta(3-2 \eta)}\right] \cdot\left[\frac{2 A}{(1-\eta)^{3}}-\frac{3(3-4 \eta)}{\eta^{2}(3-2 \eta)^{2}}\right]- \\
& \frac{9 \mu_{l}[\eta(3-2 \eta)+(2-\eta)(9-10 \eta)]}{8 \eta^{4}(3-2 \eta)^{3}} .
\end{aligned}
$$

Equating the resulting expression to zero, after a simple transformation we obtain the following expression: 


$$
\begin{aligned}
& {\left[\mathrm{A} \eta(3-2 \eta)-3(1-\eta)^{2}\right] \cdot\left[2 \mathrm{~A} \cdot \eta^{2}(3-2 \eta)^{2}+3(3-4 \eta)(1-\eta)\right] \cdot \eta-\frac{9}{8}} \\
& \frac{\mu_{l}}{\mu_{g}}\left[(3-2 \eta)^{2}-\eta\right](1-\eta)^{5}=0 .
\end{aligned}
$$

This equation expresses the analytical relationship between the true volumetric gas content $\varphi=\frac{F_{g}}{F}=(1-\eta)^{2}=0$ and the mass flow rate vapor content

$$
x=G_{r} / G .
$$

Concerning the variable $\varphi$, equation (21) will look as follows:

$[\mathrm{A} \cdot(1-\sqrt{\varphi})(1+3 \sqrt{\varphi})-3 \varphi]\left[2 \mathrm{~A} \cdot(1-\sqrt{\varphi})^{2} \cdot(1+2 \sqrt{\varphi})^{2}+3 \sqrt{\varphi} \cdot(4 \sqrt{\varphi}-1)\right](1-$ $\sqrt{\varphi})-\frac{9}{8} \cdot \frac{\mu_{l}}{\mu_{g}}\left[(1+2 \sqrt{\varphi})^{2}-(1-\sqrt{\varphi})\right] \cdot \varphi^{2} \sqrt{\varphi}=0$

We solve the problem for a parabolic velocity profile in the annual liquid layer and in a gas nucleus. In this case

$$
\begin{array}{r}
w_{l}=\frac{w_{0 l}}{\delta \cdot\left(2 R_{0}-\delta\right)} \cdot\left(R_{0}^{2}-R^{2}\right) \\
\frac{d w_{l}}{d R}=-\frac{2 w_{0 l} \cdot R}{\delta \cdot\left(2 R_{0}-\delta\right)},
\end{array}
$$

where $R_{0}-\delta \leq R \leq R_{0}$.

The average velocity of fluid in the annual layer of the liquid is equal to

$$
\begin{gathered}
w=\frac{1}{2} w_{0 l}, \\
w_{0 l}=\frac{2 G_{l}}{\pi \rho_{l} \cdot\left[R_{0}^{2}-\left(R_{0}-\delta\right)^{2}\right]}=\frac{2 G_{l}}{\pi \rho_{l} \cdot R_{0}^{2} \cdot\left[1-(1-\eta)^{2}\right]} .
\end{gathered}
$$

Second work of internal friction forces in liquid annular layer of unit length

$$
E_{l}=2 \pi \mu_{l} \int_{R_{0}-\delta}^{R_{0}} R\left(\frac{d w_{l}}{d R}\right)^{2} d R=\frac{8 \pi \mu_{l} w_{0 l}^{2}}{\delta^{2} \cdot\left(2 R_{0}-\delta\right)^{2}} \cdot \int_{R_{0}-\delta}^{R_{0}} R^{3} d R .
$$

After calculating the integral (26), and taking into account the relation(20), we obtain

$$
\mathrm{E}_{l}=\frac{8 \pi \mu_{l} w_{l}^{2} \cdot\left[1+(1-\eta)^{2}\right]}{1-(1-\eta)^{2}}
$$

Using the continuity equation

$$
\mathrm{E}_{l}=\frac{8 \pi \mu_{l} G^{2}(1-x)^{2} \cdot\left[1+(1-\eta)^{2}\right]}{\pi \rho_{l}^{2} R_{0}^{4} \eta^{3}(2-\eta)^{3}}
$$

According to formula (8) the second work of internal friction forces in a gas nucleus of unit length

$$
E_{g}=8 \pi \mu_{g}\left(\overline{w_{g(\text { абс })}}-w_{0 l}\right)^{2}=\frac{8 \pi \mu_{g} G^{2}(1-x)^{2}}{\pi \rho_{l}^{2} R_{0}^{4}} \cdot\left[\frac{\mathrm{A}}{(1-\eta)^{2}}-\frac{2}{\eta(2-\eta)}\right]^{2} .
$$

Consequently

$$
E_{g}=\frac{\delta \mu_{g} G^{2}(1-x)^{2}}{\pi \rho_{l}^{2} R_{0}^{4}} \cdot\left\{\left[\frac{\mathrm{A}}{(1-\eta)^{2}}-\frac{2}{\eta(2-\eta)}\right]+\frac{\mu_{l} \cdot\left[1+(1-\eta)^{2}\right]}{\mu_{g} \eta^{3}(2-\eta)^{3}}\right\} .
$$

In the expression (30) we replace the variable $\eta$ with another one: $(1-\eta)^{2}$, i.e. the value of the true volumetric gas content $-\varphi$. Then: 


$$
E_{g}=\frac{\delta \mu_{g} G^{2}(1-x)^{2}}{\pi \rho_{l}^{2} R_{0}^{4}} \cdot\left[\left(\frac{\mathrm{A}}{\varphi}-\frac{2}{1-\varphi}\right)^{2}+\frac{\mu_{l}}{\mu_{g}} \cdot \frac{(1+\varphi)}{(1-\varphi)^{3}}\right] .
$$

We differentiate the value $E$ with respect to the variable $\varphi$.

Equating the first derivative to zero

$$
\begin{aligned}
& \frac{\partial}{\partial \varphi}\left[\left(\frac{\mathrm{A}}{\varphi}-\frac{2}{1-\varphi}\right)^{2}+\frac{\mu_{l}}{\mu_{g}} \cdot \frac{(1+\varphi)}{(1-\varphi)^{3}}\right]=2\left(\frac{\mathrm{A}}{\varphi}-\frac{2}{1-\varphi}\right) \cdot\left[-\frac{\mathrm{A}}{\varphi^{2}}-\frac{2}{(1-\varphi)^{2}}\right]+ \\
& +\frac{\mu_{l} \cdot(1-\varphi)^{3}+3(1-\varphi)^{2}(1+\varphi)}{\mu_{g} \cdot(1-\varphi)^{4}}=\frac{2 \mu_{l}(2+\varphi)}{\mu_{g} \cdot(1-\varphi)^{4}}-2\left[\frac{\mathrm{A}}{\varphi^{2}}+\frac{2}{(1-\varphi)^{2}}\right] \cdot\left(\frac{\mathrm{A}}{\varphi}-\frac{2}{1-\varphi}\right),
\end{aligned}
$$

we obtain the following equation expressing the minimum condition for the resultant second work of internal friction forces:

$$
\begin{gathered}
{\left[\frac{\mathrm{A}}{\varphi^{2}}+\frac{2}{(1-\varphi)^{2}}\right]\left(\frac{\mathrm{A}}{\varphi}-\frac{2}{1-\varphi}\right)-\frac{\mu_{l}}{\mu_{g}} \cdot \frac{(2+\varphi)}{(1-\varphi)^{4}}=0 ;} \\
(0<\varphi<I) .
\end{gathered}
$$

Analysis of the obtained solutions (22) and (32) shows their differences. It confirms the advantage of the method of integral relations used in the problem under consideration. The method is based on one of the fundamental laws of mechanics. The solutions can also be used for the wave flow of a liquid film if the value $\eta$ means relative thickness of the liquid layer. It is also to introduce the so-called effective viscosity coefficient as a parameter that takes into account wave nature of the interface between the gas nucleus and the liquid layer $[7,8]$.

\subsection{Turbulent flow in the gas nucleus of two-phase flow, laminar flow in the annular liquid layer}

The velocity distribution over the cross section for a turbulent flow of a medium in a cylindrical channel is expressed by the formula:

$$
\frac{w_{g}}{w_{0 g}}=1-\frac{\operatorname{Arth} \sqrt{m t}-\operatorname{arctg} \sqrt{m t}}{0.805 \cdot \lg R e_{g}}
$$

where $t$ - relative distance from the nucleus axis; $t=\frac{R}{R_{0}-\delta}=\frac{R}{R_{g}} ; \operatorname{Arth} \sqrt{m}-\operatorname{arctg} \sqrt{m}=0,805 \lg R e_{g}$. For $R e_{g}>400$ the value $m$ is very close to one.

The choice of this law of velocity distribution is due not only to the fact that it very well reflects the experimental data, given the deformation of the velocity profile with a change in the Re number, and primarily because the simpler distribution laws (power law and logarithmic) used in hydrodynamics have a significant drawback in the fact that the derivative on the wall turns to infinity, which limits the possibilities of solving this and other boundary problems.

The stress of turbulent friction in accordance with the Prandtl-Karman theory is expressed here by the formula

$$
\tau_{g}=x^{2} \rho_{g}\left(R_{0}-\delta\right)^{2} \cdot\left(1-m^{2} t^{2}\right)^{2} \cdot\left(\frac{d w_{g}}{d R}\right)^{2} .
$$

Differentiating expression (33) with respect to the variable $R$, we have

$$
\frac{d w_{g}}{d R}=\frac{d w_{g}}{d t} \cdot \frac{d t}{d R}=-\frac{w_{0 g} m \sqrt{m t}}{0,805 \lg R e_{g} \cdot\left(1-m^{2} t^{2}\right) \cdot\left(R_{0}-\delta\right)}
$$

Consequently, for the tangential frictional stress in the gas nucleus, we obtain the following expression:

$$
\tau=\frac{\rho_{g} x^{2} m^{3} w_{0 g}^{2} t}{\left(0,805 \lg e_{g}\right)^{2}}
$$

The average flow rate $\bar{w}_{g}$ is determined by the following formula: 


$$
\bar{w}_{g}=\frac{2 \pi \int_{0}^{R_{0}-\delta} \rho_{g} R w_{g} d R}{\pi \rho_{g}\left(R_{0}-\delta\right)^{2}}
$$

After substituting expression (33) and calculating the integral (36), the relationship between the average and maximum flow velocities takes the following form:

$$
\frac{\bar{w}_{g}}{w_{\mathrm{o} g}}=\frac{\lg R e_{g}-0,83}{\lg R e_{g}} .
$$

Consequently, the expression for the tangential frictional stress (35) can be rewritten in the following form:

$$
\tau=\frac{x^{2} \rho_{g} m^{3} w_{g}^{2} t}{0,805^{2}\left(\lg R e_{g}-0,83\right)^{2}}=\mathrm{ct}
$$

Second work of internal friction forces in the gas nucleus is expressed by the integral

$$
\mathrm{E}_{g}=2 \pi 0 \int_{0}^{R g} \tau_{g} R \frac{d w}{d R} d R
$$

According to formulas (34) and (37)

$$
\begin{gathered}
\left|\frac{d w_{g}}{d R}\right|=\frac{c_{2} \sqrt{m t}}{1-m^{2} t^{2}}, \\
\text { where } \mathrm{C}_{2}=\frac{2 \pi \mathrm{c}_{1} \mathrm{c}_{2} R_{g}^{2}}{m^{3}} \int_{0}^{m} \frac{(m t)^{2} \sqrt{m t}}{1-(m t)^{2}} d(m t) .
\end{gathered}
$$

To calculate the integral in expression (41), we introduce the notation $\sqrt{m t}=z$.

Then $d(m t)=2 z d z$.

The required integral can be rewritten in the following form:

$$
\begin{gathered}
2 \int_{0}^{\sqrt{m}} \frac{z^{6}}{1-z} d z=2 \int_{0}^{\sqrt{m}}\left(\frac{z^{2}}{1-z^{4}}-z^{2}\right) d z=2 \int_{0}^{\sqrt{m}}\left(\frac{1}{4(1+z)}+\frac{1}{4(1-z)}-\frac{1}{2\left(1+z^{2}\right)}-z^{2}\right) d z= \\
\operatorname{Arth} \sqrt{m}-\operatorname{arctg} \sqrt{m}-\frac{2}{3}(\sqrt{m})^{3} \approx 0.805\left(\lg R e_{g}-0,83\right) ; \\
\operatorname{Re}_{g}>400 .
\end{gathered}
$$

Substituting the calculated value of the integral in expression (41), we obtain

$$
E_{g}=\frac{2 \pi x^{2} \rho_{g} \overline{w_{g}^{3}} R g}{0,805^{2}\left(\lg R e_{g}-0,83\right)^{2}}
$$

The average velocity in the gas nucleus relative to the liquid film is less than the absolute average velocity $w_{g \text { (абс) }}$ by the value $w_{\mathrm{o} l}$ :

$$
\overline{w_{g}}=\overline{w_{g \text { (абс })}}-w_{\mathrm{ol}} .
$$

According to formulas (13) and (25)

$$
\begin{aligned}
& \overline{w_{g \text { (абс })}}=\frac{G_{r}}{\pi \rho_{g} R_{0}^{2} \varphi}=\frac{G x}{\pi \rho_{g} R_{0}^{2} \varphi} ; \\
& w_{\mathrm{o} l}=2 \overline{w_{l}}=\frac{2 G_{l}}{\pi \rho_{l} R_{0}^{2}(1-\varphi)}=\frac{2 G_{l}(1-x)}{\pi \rho_{l} R_{0}^{2}(1-\varphi)} .
\end{aligned}
$$

That is why

$$
\overline{w_{g}}=\frac{G(1-x)}{\pi \cdot R_{0}^{2} \rho_{l}} \cdot\left(\frac{\mathrm{A}}{\varphi}-\frac{2}{1-\varphi}\right) .
$$

Substituting the obtained expression of the velocity (46) into the formula (43), we obtain 


$$
E_{g}=\frac{2 x^{2} \rho_{g} G_{l}^{3} \sqrt{\varphi}}{0,805^{2} \cdot \pi^{2} R_{0}^{5} \rho_{l}^{3}\left(\lg R e_{g}-0,83\right)^{2}} \cdot\left(\frac{\mathrm{A}}{\varphi}-\frac{2}{1-\varphi}\right)^{3} .
$$

According to the formula (28)

$$
\mathrm{E}_{l}=\frac{8 \mu_{l} G^{2}{ }_{l}(1+\varphi)}{\pi \rho_{l}^{2} R_{0}^{4}(1-\varphi)^{3}}
$$

Thus, the resulting dissipative characteristic of the gas-liquid flow for the studied case will be

$$
\mathrm{E}_{l}=\frac{8 \mu_{l} G^{2}(1-\chi)^{2}}{\pi \rho_{l}^{2} R_{0}^{4}} \cdot\left[\frac{C_{3} \sqrt{\varphi}}{\left(\operatorname{lgR} e_{g}-0,83\right)^{2}} \cdot\left(\frac{A}{\varphi}-\frac{2}{1-\varphi}\right)^{3}+\frac{1+\varphi}{(1-\varphi)^{3}}\right],
$$

where $\mathrm{C}_{3}=\frac{0,386 \cdot x^{2} \rho_{g} G(1-\chi)}{\pi \rho_{l} \mu_{l} R_{0}}$.

\section{Results}

From the minimum condition of this function $\left(\frac{\partial E}{\partial \varphi}=0\right)$, we obtain the following analytical relationship between the mass flow $(x)$ and the true volumetric $(\varphi)$ gas content and the twophase flow or the corresponding phase slip coefficient

$$
\begin{gathered}
\left(\frac{A}{\varphi}-\frac{2}{1-\varphi}\right)\left\{\frac{B+0,87}{2 \sqrt{\varphi} B}-3 \sqrt{\varphi}\left[\frac{A}{\varphi^{2}}-\frac{2}{(1-\varphi)^{2}}\right]\right\}+\frac{\rho_{l} B^{2}(2+\varphi)}{\rho_{g} \operatorname{Re}_{l g}(1-x)(1-\varphi)^{4}}=0 \\
0<\varphi<1 ; \quad 0<x<1 ; .
\end{gathered}
$$

In this case

$$
B=\lg \left(\frac{R e_{g(0)} x}{\sqrt{\varphi}}\right)-0,83
$$

$R e_{\Pi г}=\frac{(\rho w) \cdot d_{0}}{\mu_{g}}-$ the reduced Reynolds number for the gas; $R e_{l g}=\frac{(\rho w) \cdot d_{0}}{\mu_{l}}-$ the reduced Reynolds number for the liquid phase; $d_{0}$ - inner diameter of the pipe, $\mathrm{m}$; $(\rho w)$ - mass rate of gas-liquid flow, $\mathrm{kg} / \mathrm{m}^{2} \cdot \mathrm{s}$.

\section{Discussion}

The calculated values of $\varphi$ according to the formula (50), found from the condition of a minimum of the dissipative characteristic of the two-phase " $E$ " flow, are in good agreement with the experimental data of various authors [9-15] and are very close to the values calculated of the known dependences for the steam-water flow of Z.L. Miropol'skiy. It confirms the reliability of the physical model underlying the proposed theoretical solution and points to a new approach to this problem different from the traditional one.

A comparative analysis of the experimental data according to $\varphi$ in heated and unheated flows showed that in the range $x>0,1$ the thermal flow practically does not affect the value of the true volumetric vapor content.

Equation (50) is somewhat cumbersome for engineering calculations. However, when using a computer, the establishment between the values of $\varphi$ and $x$ for different values of other parameters of the gas-liquid flow is not difficult.

The performed mathematical analysis showed a very small effect of the logarithmic functions entering into the equation (50). It is explained by the fact that the coefficient $\left(\lg R e_{g}-0,83\right)^{2}$ entering the initial formula (49), which reflects the deformation of the velocity profile in the gas nucleus, changes insignificantly in the turbulent flow.

If, when differentiating function (49), the indicated coefficient is considered to be independent of $\varphi$, then we have 
Or

$$
\frac{\partial}{\partial \varphi}\left[c \cdot \sqrt{\varphi}\left(\frac{A}{\varphi}-\frac{2}{1-\varphi}\right)^{3}+\frac{1+\varphi}{(1-\varphi)^{3}}\right]=0
$$

$$
\begin{gathered}
\frac{2}{2 \sqrt{\varphi}}\left(\frac{A}{\varphi}-\frac{2}{1-\varphi}\right)^{2}\left[\frac{A}{\varphi}-\frac{2}{1-\varphi}-6 \varphi\left(\frac{A}{\varphi^{2}}-\frac{2}{(1-\varphi)^{2}}\right) \pm \frac{2(2+\varphi)}{(1-\varphi)^{4}}\right]=0 \\
0<\varphi<1 .
\end{gathered}
$$

\section{Conclusion}

Ffter simple transformations we obtain the following equation:

$$
\mathrm{C}[\mathrm{A}(1-\varphi)-2 \varphi]^{2}\left[5 \mathrm{~A}(1-\varphi)^{2}+2 \varphi(1+\varphi)\right]-4 \varphi^{3} \sqrt{\varphi}(2+\varphi)=0 .
$$

In this case

$$
\mathrm{C}=\frac{0,193 \aleph^{2} \rho_{g} R e_{l g}(1-x)}{\pi \rho_{l}\left(\lg R e_{g}-0,83\right)} .
$$

The disadvantage of equations (32), (50) and (53) is that they express the dependence in implicit form.

$$
\varphi=f\left(x, \rho_{l} / \rho_{g}, \mu_{l} / \mu_{g}, \rho w\right) .
$$

For engineering calculations, these equations can be substantially simplified. Since the velocity at the boundary between the gas and liquid phases for the values $x>0,1$ is much less than the absolute velocity of the gas phase, then with sufficient accuracy for practical calculations it can be assumed that

$$
w_{0 g}=k_{1} \overline{w_{g}},
$$

where $k_{1}$ - averaged coefficient of proportionality.

$$
w_{0 g}=\frac{k_{1} \cdot G \cdot x}{\varphi \rho_{g}}=\frac{k_{1}(\rho w) x}{\varphi \rho_{g}} .
$$

Therefore, the expression for the dissipation energy (31) for a two-phase flow can be written in the following form:

$$
E=\frac{8 \pi \mu_{g}(\rho w)^{2}(1-x)^{2}}{\rho_{l}^{2}}\left[\frac{k_{2} A^{2}}{\varphi^{2}}+\frac{\mu_{l}(1+\varphi)}{\mu_{g}(1-\varphi)^{3}}\right] .
$$

From the condition for the minimum of this function (with respect to the variable $\varphi$ ), we obtain the following expression:

$$
\frac{(2+\varphi) \varphi^{3}}{(1-\varphi)^{4}}=\frac{k_{2} A \mu_{g}}{\mu_{l}}
$$

The left side of equation (58) is well approximated by expression

Therefore, we can write

$$
7,1 \cdot\left(\frac{\varphi}{1-\varphi}\right)^{3,6} \text {. }
$$

$$
\begin{aligned}
\frac{\varphi}{1-\varphi} & =\left(\frac{k_{2} A \mu_{g}}{7,1 \mu_{l}}\right)^{0,278} . \\
\text { Then } \varphi & =\frac{1}{1+k \cdot\left(\frac{\mu_{l}}{A^{2} \mu_{g}}\right)^{0,278}},
\end{aligned}
$$

where $\mathrm{A}=\frac{x}{1-x} \cdot \frac{\rho_{l}}{\rho_{g}}$.

By choosing the appropriate value of the coefficient $k$, we can take into account not only the character of the velocity distribution over the cross section, but also the change in the 
parameters $\rho_{l} / \rho_{g}$ and $\mu_{l} / \mu_{g}$ in the result of mechanical carryover of liquid particles into the gas nucleus, and also "loosening" of the liquid annular layer with intensive vaporization in steam generating pipes.

Equation (60) satisfactorily generalizes the experimental data on the steam-water flow at a value of $k=0.5$.

In accordance with formulas (44), (45) and (60), we obtain the following expression for the slip coefficient:

$$
S=k\left[\frac{x \rho_{l}}{(1-x) \rho_{g}}\right]^{0,444}\left(\frac{\mu_{l}}{\mu_{g}}\right)^{0,278}
$$

The problem of the distribution of the phases of a gas-liquid flow in plane channels is very interesting. The theory considered allows us to investigate this question.

Since the main purpose of this work was to study the flow of two-phase flows in pipes, we give only two formulas obtained for determining the true gas volume content $\varphi$ and the slip coefficient in flat channels

$k=0,5$.

$$
\begin{gathered}
\varphi=\frac{1}{1+\frac{k(1-x) \rho_{g}}{x \rho_{l}} \sqrt{\frac{\mu_{l}}{\mu_{g}}}} ; \\
S=k \sqrt{\frac{\mu_{l}}{\mu_{g}}}
\end{gathered}
$$

The advantage of the obtained equations and formulas for determining the true volumetric vapor content, in contrast to the known ones, is that they reflect the influence of the viscous characteristics of the gas or liquid media and can be used to calculate the vapor-liquid and gas-liquid flows of various components.

The latter is especially important for the study of many technological processes taking place in various apparatuses of the chemical industry, refrigeration and heat power engineering.

\section{References}

1. H. G., Vassilicos Ch. (eds.) Prediction of Turbulent Flows. (Cambridge University Press, 2005)

2. G.A. Dreytser. Efficiency of the use of flow swirls for intensification of flow in tubular heat exchangers. Moscow (2005).

3. I.R. Prigozhin. Introduction to the thermodynamics of irreversible processes. Izhevsk, 160 (2001).

4. I.R. Prigozhin, D. Kondepudi. Modern thermodynamics. From heat engines to dissipative structures. Moscow, 44 (2002).

5. G. Nikolis. Cognition of complex things. Moscow, 345 (2012).

6. B. Palm Heat transfer in heat exchangers designer for minimum charge. In: IIR//IIF. Stockholm, 5, 84-89 (2002).

7. S.E. Tarasevich, A.B. Yakovlev. On the boundary of the dispersed-annular flow regime in channels with continuous twisting. (Moscow; Novosibirsk, 2004).

8. Bandara Filho Enio P., Saiz Jabardo Jose. Int. J. Refrig., 8, 895-903 (2004).

9. V. Soldo, T. Curko, and M. Grozdek. Transfer from Carnot to Lorenz process using zeotropic mixtures. In: IIR/IIF. Stockholm, 63-66 (2002).

10. G.A. Dreytser. Efficiency of the use of flow swirls for intensification of flow in tubular heat exchangers. Moscow (2005). 
11. Yu.M. Brodov, A.Yu. Ryabchikov, and K.E. Aronson. Investigation of a number of methods for intensifying heat transfer in energy heat exchangers, 6, 49 (2002).

12. V.V. Yagov. Heat transfer and crises during boiling in swirling flows. Moscow (2005).

13. A. Klaszak Heat and mass transfer, 37, 443-448 (2001).

14. Yu.I. Shanin. Heat transfer when the flow is twisted in a square channel. (Moscow, 2005).

15. V.G. Bukin, A.Yu. Kuzmin. Refrigeration machines operating on non-azeotropic refrigerant mixtures (Astrakhan, 2003). 